\title{
Schutzkonzepte in der Pflegekinderhilfe
}

\author{
Reformbedarfe zur Verwirklichung
}

Am 20. November 2019 feierte die UN-Kinderrechtskonvention ihr 30-jähriges Bestehen. In ihr ist u. a. festgehalten, dass alle Kinder und Jugendlichen ein Recht auf körperliche Unversehrtheit, Gleichbehandlung, Beteiligung, Beschwerde und Anhörung haben. Seit gut zehn Jahren werden in vielen Organisationen (KiTas, Schulen, Sportvereine, Jugendzentren, Einrichtungen der stationären Erziehungshilfe, Kliniken) mittlerweile Schutzkonzepte gegen sexualisierte Gewalt etabliert.

S chutzkonzepte sind somit „ein bedeutsamer Teil gesellschaftlicher Bemühungen, Kinderrechte zu stärken und den Kinderschutz zu verbessern “ (Kappler et al. 2019, S. 13). Ein Bereich der bis dato jedoch in diesem Zusammenhang wenig im Blick war, ist die Pflegekinderhilfe. Die Entwicklung und Implementierung von Schutzkonzepten in der Infrastruktur der Pflegekinderhilfe ist somit dringend geboten und hoch aktuell.

\section{Schutzkonzepte zur Stärkung und Wahrung persönlicher Rechte}

Schutzkonzepte vereinen abgestimmte Vorgehen bzw. Verfahren, die darauf abzielen, Kinder und Jugendliche durch Maßnahmen der Sensibilisierung, der Prävention,

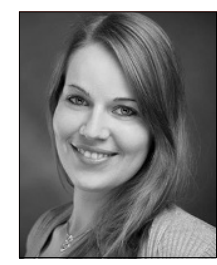

\section{Laura Husmann}

Hildesheim, Deutschland

*1988, Sozial- und Organisationspädagogin M.A., seit 2017 wissenschaftliche Mitarbeiterin am Institut für Sozial- und Organisationspädagogik, Universität Hildesheim.

husmann!@uni-hildesheim.de

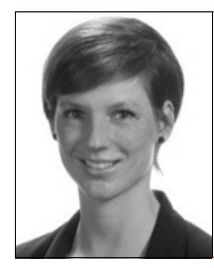

Tanja Rusack

Hildesheim, Deutschland

*1984, Dr., Diplom-Pädagogin, seit 2013 wissenschaftliche Mitarbeiterin am Institut für Sozial- und Organisationspädagogik, Universität Hildesheim.

rusack@uni-hildesheim.de

Zusammenfassung Im Forschungsprojekt „FosterCare“ werden im Hinblick auf die Entwicklung und Implementierung von Schutzkonzepten in der Pflegekinderhilfe und die Verwirklichung von Rechten junger Menschen in Pflegefamilien Reformbedarfe in der Kinder- und Jugendhilfe aufgezeigt.

Schlüsselwörter Schutz, Schutzkonzepte, Pflegekinderhilfe, Kinder- und Jugendrechte, Gesetzesreformen der Intervention und der langfristigen Aufarbeitung vor Gewalt und Machtmissbrauch durch Erwachsene und Peers besser zu schützen (RTSKM 2011). Diesem Verständnis nach geht es bei einem Schutzkonzept zunächst darum, sich zu vergegenwärtigen, wo und wie es dazu kommen kann, dass die persönlichen Rechte von jungen Menschen aus dem Blick geraten können (Fegert et al. 2017; Kampert et al. 2020). Schutzkonzepte dienen also vor allem dazu, die Achtsamkeit einer Organisation für die Menschen-, Kinder-, Jugend- und Sozialrechte von anvertrauten Schutzbefohlenen zu erhöhen.

Bei einem Schutzkonzept handelt es sich demnach um einen partizipativen Organisationsentwicklungsprozess, der Prozesse der selbstkritischen Analyse von Organisationen, der Planung passfähiger Prävention, der Intervention in Verdachtsfällen sowie der Aufarbeitung von möglichen Fällen des Machtmissbrauchs beinhaltet. In diese Prozesse sind alle Akteur_innen (junger Mensch, haupt- und ehrenamtliche Mitarbeiter_innen, Eltern, Leitungskräfte, Behörden, Verbände etc.) mit einzubinden (Kampert et al. 2020; Wolff et al. 2017). Auf diese Weise erhöht sich die Chance, dass potentielle Schwachstellen, Modifizierungsbedarfe, aber auch Potentiale in einer Einrichtung erkannt werden und ein Schutzkonzept nicht an den Bedürfnissen der Akteur_innen vorbeiführt (Fegert et al. 2017). Schutzkonzepte werden als Seismographen für die Achtung unveräußerlicher persönlicher Rechte (Team FosterCare 2020) von jungen Menschen in Organisationen diskutiert und sind damit als andauernde und kontinuierliche Reflexionsprozesse im Rahmen einer partizipativen Organisationsentwicklung zu verstehen.

Es wird jedoch deutlich, dass Schutzkonzepte nach dieser Definition - welche sich auf Organisationen bezieht - nicht ohne weiteres auf den Bereich der Pflegekinderhilfe zu übertragen sind. Pflegefamilien sind keine Or- 


\section{Extrablick: Aktuelle Entwicklungen in der Pflegekinderhilfe}

ganisationen. Das bedeutet, „(...) dass Schutzkonzepte im Pflegekinderwesen stärker auf ein Geflecht von Infrastruktur und Familien transferiert werden müssen. Diesbezüglich existieren bisher aber kaum systematische Konzepte“ (Husmann et al. 2019, S. 44).

\section{Pflegekinderhilfe im Spannungsverhältnis}

Bislang wurden organisationale Verfahren des Kinder- und Jugendschutzes in der Pflegekinderhilfe kaum genauer betrachtet. Ein Erklärungsversuch liegt darin begründet, dass die Infrastrukturen, die die Pflegekinderhilfe gestalten, bislang nicht insgesamt im Fokus der Forschung und des Fachdiskurses zu finden waren (van Santen et al. 2019). Vielmehr stand bisher die Pflegefamilie als solche im Mittelpunkt. Erst seit einigen Jahren gibt es eine Öffnung dieser Diskussion und es wird die gesamte Infrastruktur mit ihren unterschiedlichen Akteur_innen (Pflegeelternvereine, Jugendamt, den unterschiedlichen Elternschaften, Vormundschaften, etc.) berücksichtigt (Wolf 2014).

Die Pflegekinderhilfe nach $\mathbb{3} 3$ SGB VIII (sowie auch im Rahmen der Eingliederungshilfe für junge Menschen mit seelischer Behinderung nach $\$ 35$ a SGB VIII oder als Hilfe für junge Volljährige nach $\mathbb{\$} 41$ SGB VIII) bewegt sich in einem generellen Spannungsfeld. Sie gehört zum einen neben der Heimerziehung nach $\$ 34$ SGB VIII zu den Hilfen zur Erziehung, zum anderen wird sie als zivilgesellschaftliches Engagement verstanden und betrifft somit besonders schützenswerte Rechte einer Familie. So regelt Artikel 6 im Grundgesetz das schützenswerte Gut der „Familie“ und stellt diese damit unter besonderen Schutz. Aufgrund dieser Sonderstellung der Pflegekinderhilfe können Konzepte, wie sie bspw. in der Heimerziehung entwickelt wurden, nicht einfach übertragen werden. Auch Auflagen können schwerer initiiert werden, wodurch eine Pflicht zur Einrichtung eines Beschwerdemanagements, z. B. wie in $\$ 45$ SGB VIII für Einrichtungen der Heimerziehung verankert, im Kontext der Pflegekinderhilfe bspw. nicht gilt.

\section{Gesetzlicher Reformbedarf zur Absicherung von Schutzkonzepten}

Das interdisziplinäre, vom Bundesministerium für Bildung und Forschung (BMBF) von 2018-2020 geförderte Forschungsprojekt „FosterCare - Rechte Stärken. Beteiligen. Schützen. Junge Menschen in Pflegefamilien " von der Universität Hildesheim, dem Universitätsklinikum Ulm und der Hochschule Landshut (www.fostercare.de), widmet sich intensiv der Frage, wie die höchstpersönlichen Rechte von jungen Menschen in Pflegefamilien gestärkt und geschützt werden können. Ziel des Projektes ist es, die Schutz- und Betei- ligungsrechte von jungen Menschen in Pflegefamilien zu verbessern und damit zur Stärkung ihrer persönlichen Rechte beizutragen. Ausgehend von den Perspektiven und Erfahrungen von jungen Menschen in Pflegefamilien, Careleavern, Pflegeltern sowie Fachkräften werden gemeinsam mit ihnen handlungsleitende Impulse für Schutzkonzepte in der Pflegekinderhilfe erarbeitet, um daraus Empfehlungen für die Fachpraxis und Fachpolitik ableiten zu können. In diesem Zusammenhang wurden die rechtlichen Grundlagen des SGB VIII in Bezug auf die Pflegekinderhilfe analysiert und mit Expert_innen und unterschiedlichen Akteur_innen reflektiert.

So sind aus dem Projekt heraus die folgenden Anpassungen in Bezug auf die Pflegekinderhilfe im Kontext der SGB VIII-Reform vorzunehmen. Es geht hier auch um eine Anpassung im Recht, da Schutzkonzepte nicht nur in der Heimerziehung oder anderen einrichtungsbezogenen Strukturen zentral sind, sondern auch die Infrastruktur der Vollzeitpflege aus einer kinderrechtlichen Perspektive qualifiziert werden muss. Es ist klar, dass dabei einige Forderungen nicht neu sind, sie müssen aber in der Pflegekinderhilfe aus einer kinderrechtlichen Perspektive neu gewichtet werden (s. Infobox 1).

\section{Ausblick: Qualitätsstandards in der Infrastruktur}

Die Kinder- und Jugendhilfe ist ein Handlungsfeld, in dem jungen Menschen zur gesellschaftlichen Teilhabe verholfen wird und Entwicklungschancen ermöglicht werden. Junge Menschen entdecken in Ferienlagern ihre Talente, lernen in Beratungsstellen, mit ihren Familien achtsamer umzugehen, lernen in Jugendwohngruppen die Unterstützungspotentiale einer Gruppe kennen oder erfahren in einer Pflegefamilie die Förderung, die ihnen zusteht. Seit der öffentlichen Thematisierung von Missbrauchsskandalen - auch in der Pflegekinderhilfe - ist aber auch deutlich geworden, dass Kinder und Jugendliche in diesen Kontexten ebenso Unrecht und Missbrauch erfahren können. So muss eingeräumt werden, dass auch die Infrastrukturen der Pflegekinderhilfe wie alle anderen sozialen Orte auch - nicht per se sichere Orte sind. Dieser Entzauberung des Schutzes sind alle Handlungsfelder unterworfen. Junge Menschen benötigen darum an allen sozialen Orten des Heranwachsens und der Förderung eine starke Lobby zur Entwicklung von Schutzkonzepten zur Verwirklichung ihrer persönlichen Rechte auf Schutz, Beteiligung und Beschwerde. Die Pflegekinderhilfe ist davon nicht ausgenommen. In der Pflegekinderhilfe sind Schutzkonzepte nicht nur auf den als gelebten (Familien-) Alltag zu begrenzen, sondern in Verantwortung der Infrastruktur der Pflegekinderhilfe zu verorten: es geht dabei nicht nur um 


\section{Infobox 1}

- Es ist z. B. in $\$ 37$ SGB VIII festzuhalten, dass es die Verantwortung der Jugendämter ist, Schutzkonzepte für die Infrastruktur der Pflegekinderhilfe zu entwickeln. Indem die Pflegekinderhilfe explizit in \$ 79a SGB VIII aufgenommen wird, ist dafür Sorge zu tragen, dass Schutzkonzepte im Rahmen der Qualitätsentwicklung fortgeschrieben und evaluiert werden.

- In $\mathbb{S} \mathbb{3} 33 ; 44 ; 45$ SGB VIII ist festzuhalten, dass die Pflegefamilien in den Kontext der Schutzkonzepte eingebunden sind - mindestens Beschwerdeverfahren für die jungen Menschen in der Infrastruktur vorhanden sein müssen - und die Pflegeeltern in den Auswahlverfahren entsprechend informiert und qualifiziert werden müssen. Zudem ist den Pflegeeltern ein Beratungsrecht in $\mathbb{S} 8 \mathrm{~b}$ SGB VIII einzuräumen.

- In $\mathbb{2} 27$ SGB VIII ist jungen Menschen bei einer Fremdplatzierung ein eigenständiges Interessenvertretungsrecht einzuräumen und abzusichern. In $\ 36$ SGB VIII ist festzuhalten, dass die jungen Menschen in Hilfeplangesprächen über ihre Rechte regelmäßig und altersgerecht informiert werden. Es muss sichergestellt werden, dass die jungen Menschen Beschwerdeverfahren und - soweit vorhanden - Ombudsstellen kennen oder/und ihnen eine kontinuierliche Ansprechperson zur Verfügung gestellt wird, die von den jungen Menschen ausgewählt werden kann. Weiterhin ist es für die jungen Menschen, die in Pflegefamilien aufwachsen, grundlegend, dass z. B. in $\$ 37$ Abs. 2 SGB VIII festgeschrieben wird, dass sie Unterstützung in der Organisation von kollektiven Formen der Selbstvertretung erhalten müssen.

Schließlich wäre es insgesamt für die Stärkung der Kinderrechte - auch über die Pflegekinderhilfe hinaus - ein wichtiges Signal, wenn $\mathbb{1} 1$ Abs. 3 SGB VIII nicht als „soll“, sondern als uneingeschränkte Verpflichtung formuliert wäre, die nicht primär auf Institutionen beschränkt ist.

Auszug aus: „POSITIONEN Kinderrechte in der Vollzeitpflege - Reformbedarf zur Verwirklichung von Schutzkonzepten in der Infrastruktur der Pflegekinderhilfe" (Team FosterCare 2020). ein Konzept, sondern um Schutzprozesse, deren Inhalte im Alltäglichen Einzug finden, gemeinsam gelebt, stetig überprüft und neu ausgerichtet werden sollten.

Funding. Open Access funding provided by Projekt DEAL.

Open Access. Dieser Artikel wird unter der Creative Commons Namensnennung 4.0 International Lizenz veröffentlicht, welche die Nutzung, Vervielfältigung, Bearbeitung, Verbreitung und Wiedergabe in jeglichem Medium und Format erlaubt, sofern Sie den/die ursprünglichen Autor(en) und die Quelle ordnungsgemäß nennen, einen Link zur Creative Commons Lizenz beifügen und angeben, ob Änderungen vorgenommen wurden.

Die in diesem Artikel enthaltenen Bilder und sonstiges Drittmaterial unterliegen ebenfalls der genannten Creative Commons Lizenz, sofern sich aus der Abbildungslegende nichts anderes ergibt. Sofern das betreffende Material nicht unter der genannten Creative Commons Lizenz steht und die betreffende Handlung nicht nach gesetzlichen Vorschriften erlaubt ist, ist für die oben aufgeführten Weiterverwendungen des Materials die Einwilligung des jeweiligen Rechteinhabers einzuholen.

Weitere Details zur Lizenz entnehmen Sie bitte der Lizenzinformation auf http://creativecommons.org/licenses/by/4.0/deed.de.

\section{Literatur}

Fegert, J. M., Schröer, W., \& Wolff, M. (2017). Persönliche Rechte von Kindern und Jugendlichen. Schutzkonzepte als organisationale Herausforderung. In M. Wolff, W. Schröer \& J. M. Fegert (Hrsg.), Schutzkonzepte in Theorie und Praxis. Ein beteiligungsorientiertes Werkbuch (S. 14-24). Weinheim, Basel: Beltz Juventa.

Husmann, L., Rusack, T., \& Schröer, W. (2019). Rechte Stärken. Beteiligen. Schützen - Schutzkonzepte mit jungen Menschen in Pflegekinderhilfe entwickeln. FamilienBande, 01/2019, 43-47.

Kampert, M., Wolff, M., \& Schröer, W. (2020). Schutzkonzepte und Gefährdungsanalysen zur Herstellung einer Kultur der Achtsamkeit in Organisationen. In M. Kampert, et al. (Hrsg.), Lehrbuch Schutzkonzepte und Diversität in Organisationen gestalten. Fokus: Junge Menschen mit Fluchterfahrung (S. 90-104). Weinheim, Basel: Beltz Juventa.

Kappler, S., et al. (2019). Kinder und Jugendliche besser schützen - der Anfang ist gemacht. Schutzkonzepte gegen sexuelle Gewalt in den Bereichen: Bildung und Erziehung, Gesundheit, Freizeit. Berlin: Arbeitsstab des Unabhängigen Beauftragen für Fragen des sexuellen Kindesmissbrauchs.

Runder Tisch Sexueller Kindesmissbrauch (RTSKM) (2011). Abschlussbericht. Sexueller Kindesmissbrauch in Abhängigkeits- und Machtverhältnissen in privaten und öffentlichen Einrichtungen und im familiären Bereich. Berlin: Bundesministerium für Justiz (BMJ), Bundesministerium für Familie, Senioren, Frauen und Jugend (BMFSFJ) \& Bundesministerium für Bildung und Forschung (BMBF).

van Santen, E., Pluto, L., \& Peucker, C. (2019). Pflegekinderhilfe - Situation und Perspektiven. Empirische Befunde zu Strukturen, Aufgabenwahrnehmung sowie Inanspruchnahme. Weinheim, Basel: Beltz Juventa.

Team FosterCare (2020). POSITIONEN Kinderrechte in der Vollzeitpflege - Reformbedarf zur Verwirklichung von Schutzkonzepten in der Infrastruktur der Pflegekinderhilfe. Hildesheim: Team FosterCare. https:// doi.org/10.18442/080.

Wolf, K. (2014). Sind Pflegefamilien Familien oder Organisationen? In A. Kuhls, J. Glaum \& W. Schröer (Hrsg.), Pflegekinderhilfe im Aufbruch. Aktuelle Entwicklungen und neue Herausforderungen in der Vollzeitpflege (S. 74-91). Weinheim und Basel: Beltz Juventa.

Wolff, M., Schröer, W., \& Fegert, J. M. (2017). Schutzkonzepte in Theorie und Praxis. Ein beteiligungsorientiertes Werkbuch. Weinheim,Basel: Beltz Juventa. 